\title{
Positive and negative transfer of control: Instrumental response mediation and response competition
}

\author{
SHINKEN NAITOH and ARTHUR W. STAATS \\ University of Hawaii, Honolulu, Hawaii 96822
}

\begin{abstract}
Two appetitive transfer-of-control experiments were conducted to investigate the role of instrumental response learning during the noncontingent pairing phase in transfer. The behavioral observation made in Experiment 1 suggested the operation of a food-cup approach response mediation. When the suggested food-cup approach response mediation was precluded in Experiment 2, a negative transfer effect was obtained. The two experiments exposed methodological problems involved in appetitive transfer experiments and raised questions with regard to the validity of the conclusions drawn in the past based on the type of transfer experiments reported here.
\end{abstract}

Although the results from transfer-of-control experiments are taken to reflect the effect of some noninstrumental response mediator learned in the noncontingent pairing phase of the experiment (see Trapold \& Overmier, 1972), it is generally accepted that some instrumental response, such as a food-cup approach response (FCAR), is also learned in the pairing phase. The discussion of instrumental response learning during the pairing phase typically has concerned the possibility of instrumental mediation. However, both Carlson (1976) and Flaherty and Davenport (1968) mention that the FCAR learned to the conditioned stimulus (CS) by the experimental animals may be competing with the barpress response in the testing phase. The response latency measure commonly used in transfer experiments would be expected to be sensitive to such a competing response tendency. It was thus reasoned that since the transfer effect is observed in spite of the competing response, the transfer effect must be quite robust.

In beginning the analysis of transfer of control within the framework of the three-function learning theory of social behaviorism (see Staats, 1975), the purpose of Experiment 1 was to validate the transfer phenomenon by replicating the prototypical appetitive transfer experiment. Previous social behaviorism research has shown the importance of analyzing the stimuli and responses in experimental situations (Staats, 1964). In addition to the usual dependent measure of response latency, therefore, this was done during the entire transfer testing phase, to make explicit what actually happens

These studies were conducted by the first author as part of his master's thesis under the supervision of the second author. Requests for reprints should be sent to Arthur W. Staats, Department of Psychology, University of Hawaii, Honolulu, Hawaii 96822. in a usual transfer experiment. Such detailed observation might be expected to reveal any possible competing or facilitating instrumental responses.

\section{EXPERIMENT 1}

\section{Method}

Subjects and Apparatus. Eight naive male Long-Evans hooded rats, approximately 120 days old at the start of the study, were maintained at $80 \%$ of free-feeding body weight. One conditioning chamber $(25.3 \times 21.6 \times 20.0 \mathrm{~cm})$ with a retractable response bar located on the center of one panel $3.0 \mathrm{~cm}$ above the grid floor and a food cup $5.0 \mathrm{~cm}$ to the left of it was used. The conditioning chamber was in a sound-attenuating cubicle with a peephole, fan, and a loudspeaker. Continuous white noise inside the chamber masked control and recording equipment sounds from an adjacent room.

Procedure. All subjects were first magazine and barpress trained. Next, with response bar retracted, a 3-sec tone was presented 30 times on a noncontingent VT 60 -sec schedule during each of five sessions of the pairing phase. For experimental subjects $(n=4)$, the tone was followed by a delivery of one food pellet (Noyes, $45 \mathrm{mg}$ ); for controls $(n=4)$, food was delivered on an independent VT $60-\mathrm{sec}$ schedule, except that no two events could follow each other by less than $7.5 \mathrm{sec}$.

In the transfer testing phase, 30 trials of discrete-trial instrumental discrimination training to a tone discriminative stimulus (DS) were conducted in one session. Tone was presented on a VT 60 -sec schedule. The first barpress after tone onset delivered a food pellet and turned off the tone. Any barpress within $10 \mathrm{sec}$ prior to the scheduled onset of the tone postponed the tone onset for $10 \mathrm{sec}$. The time from the tone onset to first barpress defined response latency. Subjects' behavior was observed during this phase.

\section{Results and Discussion}

The analyses involved an analysis of variance with group and block factors, using log-transformed mean response latency data, with $\alpha$ set at .05 . Although the group effect was not significant, the Group by Block interaction was $[\mathrm{F}(2,12)=12.02, \mathrm{p}<.005]$ (see Fig- 


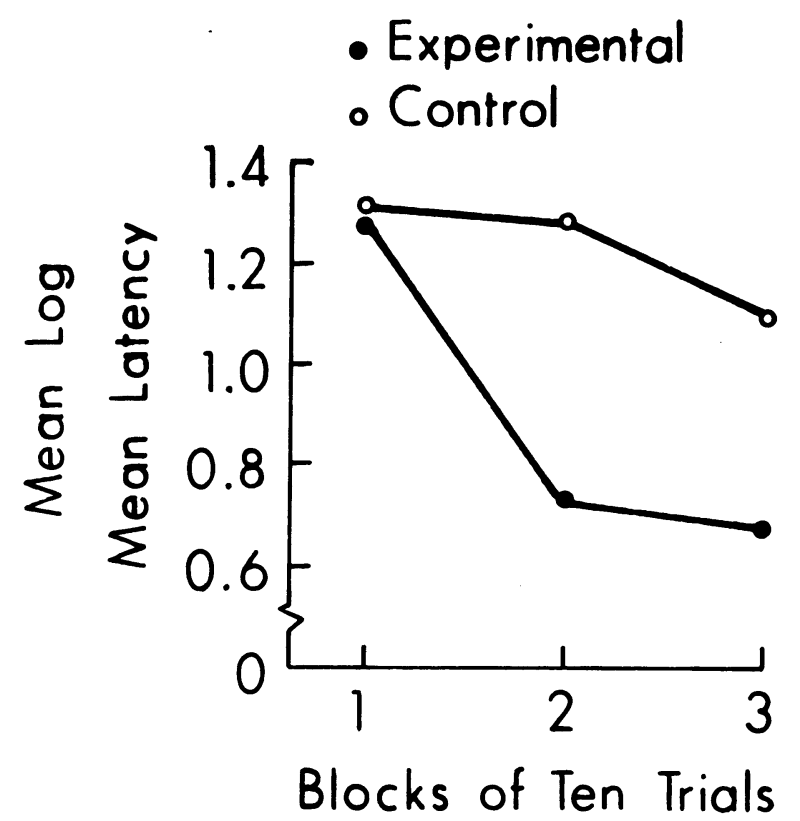

Figure 1. The log-transformed mean response latency to tone DS for the experimental and control groups over 30 trials of the test phase in three blocks of 10 trials.

ure 1). Subsequent multiple $t$ tests showed that mean latencies for the experimental group were significantly shorter than the control group at Blocks $2[\mathrm{t}(18)=3.32$, $\mathrm{p}<.05]$ and $3[\mathrm{t}(18)=2.50, \mathrm{p}<.05]$.

Although mean latencies for the first 10 trials were similar between the two groups, behavioral observation showed marked differences in response sequences at the tone onset for those 10 trials. For experimental subjects, the sequence of responses at tone onset was (1) immediate termination of any ongoing behavior, (2) FCAR and burying of the head in the food cup, and (3) barpress directly after removing the head from the food cup or starting to groom, explore, and so on, and then back to the FCAR. Experimental animal latency decrease over the trials mainly was due to decrease in the time spent at the food cup after the tone onset. The same response sequence continued throughout the test phase for all experimental animals.

In contrst, for the control animals, there was no set sequence of responses after the tone onset. However, between Trial 5 and Trial 15, tone onset began to elicit the FCAR. This FCAR produced an increase in the second trial block mean latencies for two control animals. By the end of the second block, all control subjects responded to tone onset with the response sequence observed with the experimental subjects. However, the time elapsed between the tone onset and the termination of the ongoing behavior and the time spent at the food cup were more variable from trial to trial for the control subjects.

The present experiment provided nonoperant behavioral observations (using methods called experimentalnaturalistic research; Staats, 1968) indicating the difference between the control and experimental subjects.
That is, all animals learned in barpress training the sequence of pressing the bar, approaching the food cup, consuming food, seeing the empty food cup, then returning to press the bar. The experimental animals then also learned to go to the food cup to the tone, whereas the control animals did not. These observations explain the results obtained. During the first trial block, the experimental and control animals were not different in terms of the response latencies to the tone. Although the experimental animals had the CS-food pairings, their transfer discrimination performance was not improved because the tone elicited a persistent FCAR, interfering with the barpress response. The control animals, having no response to the tone, performed equally as well at this point. The experimental animals' performance increased rapidly because the tone did initiate the chain, and it was a small matter to decrease the persistent head-in-empty-cup response.

From a general methodological point of view, reliance on the operant barpress as the sole type of data can obscure the behavioral repertoires the animals bring to that situation, rather than reveal them. It is suggested that experimental-naturalistic behavioral observation can be fruitfully employed in studying animal learning.

In conclusion, the experimental-naturalistic behavioral observation revealed that a FCAR acquired by the experimental animals may not enhance their performance in one stage of the transfer discrimination task, whereas in another stage the FCAR may be helpful. It may be noted that although some form of noninstrumental response mediator may have been acquired in the pairing phase, the effect of such a mediator would be confounded with the effect of the instrumental FCAR mediation. Experiment 2 was conducted, in part, to elucidate the role of the FCAR mediation in an appetitive transfer experiment.

\section{EXPERIMENT 2}

Staats and Warren (1974) stated that transfer would not occur if the animals had not previously learned to barpress for food: Without that training, the mediational associations between the mediator and the barpressing would not be formed. (They discussed an emotionresponse mediator, but the same would pertain to the FCAR mediation.) In the present experiment, utilizing this analysis and the Experiment 1 analysis, the effect on transfer when the established "mediating" response has no mediational associations was studied. In Experiment 2, animals were first barpress trained on CRF for water delivered into a trough to the right of the response bar. In the tone-food pairing phase, food was delivered into the food cup to the left of the response bar. In the testing phase, identical to Experiment 1, tone would elicit a FCAR which, unlike in the previous experiment, was not a mediating response for the barpress response. The FCAR was thus a competing response for the experimental animals, expected to result in a perfor- 
mance decrement. The control animals without FCAR in their response to tone would not have that decrement at the beginning.

The motivational theory (Rescorla \& Solomon, 1967), the associative theory (Trapold \& Overmier, 1972), and the revised associative theory (Overmier \& Lawry, Note 1), although based on different grounds, would all predict better performance for the experimental group, or have nothing to predict. The purpose of this experiment, however, was not theoretical criticism. It was to expose methodological problems in this type of transfer experiment and to question interpretations obtained in the transfer experiments using procedures similar to the one used here.

\section{Method}

Subjects and Apparatus. Ten naive male albino served, initially approximately 120 days old. Experiment 1 equipment was used, except for the installation of a solenoid-operated water dispenser emptying into a trough $5.0 \mathrm{~cm}$ to the right of the response bar. Also, two layers of egg cartons surrounded the cubicle to help mask the noise of the control and recording equipment, now located in the same room.

Procedure. Two days prior to the beginning of the dipper training, all subjects were put on a $23.5 \mathrm{~h}$ water-deprivation schedule with free food access.

Dipper training. With the response bar retracted, 50 deliveries of $.1 \mathrm{ml}$ of water were made on a VT 60 -sec schedule during each of two sessions. Home cage water was not available during this or the barpress training phases.

Barpress training. Barpress reinforcement was delivery of $.1 \mathrm{ml}$ of water for each of 60 responses in each of three sessions. One animal failed to reach the criterion and was replaced by another.

After the barpress training, all subjects had free water access, but they were put on a food-deprivation schedule that maintained them at $80 \%$ of free-feeding body weight.

Magazine training. With the response bar retracted, 50 food pellets were delivered during each of two sessions on a VT 60 -sec schedule. After the magazine training, the subjects were randomly assigned to two groups.

Pairing phase. The tone-food pairing phase for experimental and control animals was identical to that conducted in Experiment 1.

Transfer testing phase. Fifty trials of Experiment 1-type discrete-trial instrumental discrimination training to the tone for food reinforcement were conducted daily for three sessions. The Test Day 1 data were to be analyzed separately. Subjects' behavior was observed during this phase.

\section{Results}

An analysis of variance of the Day 1 test-phase logtransformed mean latency data was conducted, with group and block as factors. The group effect was significant $[F(1,8)=6.13, p<.05]$, with the control group responding consistently with shorter latencies. The block effect was also significant $[F(4,32)=41.50, p<.0001]$; the Group by Block interaction was nonsignificant.

An analysis of variance of the entire test-phase logtransformed mean latency data was conducted, with group, block, and day as factors. Although the group effect was nonsignificant, the Group by Day interaction was significant $[\mathrm{F}(2,16)=4.77, \mathrm{p}<.05]$ (see Figure 2). Subsequent multiple $t$ tests showed the decrease in mean

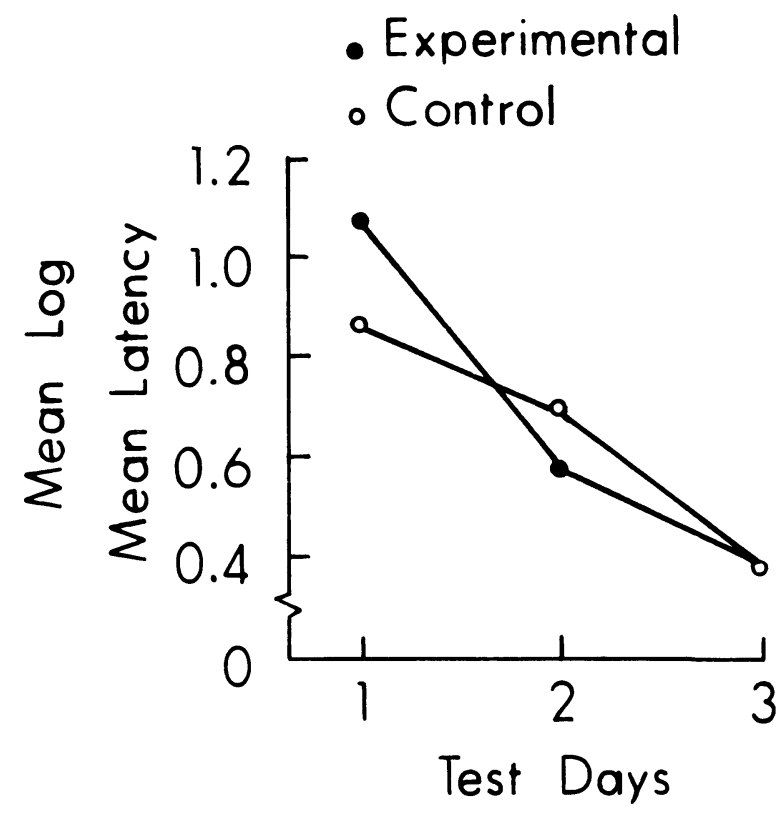

Figure 2. The log-transformed mean response latency to tone DS for the experimental and control groups over the 3 test days.

latency from Day 1 to Day 2 was significant for the experimental group $[t(16)=2.75, p<.03]$, not for the control group. All other group factor interactions were nonsignificant $(F<1)$. The block and day effects and the Day by Block interaction were significant (all $\mathrm{p}<.0001)$.

Experimental-naturalistic behavioral observation.

Day 1. All subjects stayed within approximately $5.0 \mathrm{~cm}$ of the food cup and the response bar. Experimental animals approached the food cup at tone onset, inserting their heads, or they remained in that position if there when the tone sounded. The control animals, at the tone onset, simply continued with whatever activity was ongoing. However, if they had their heads in the food cup at the time of the tone onset, they withdrew their heads from it. By the end of Test Day 1, all subjects were responding to the tone by approaching the food cup.

Day 2 and Day 3. By the end of Test Day 2, all subjects assumed a position between the food cup and the response bar, and at the tone onset, all subjects approached the food cup, inserted the head in the food cup, and barpressed without removing the head from the food cup.

\section{GENERAL DISCUSSION}

As predicted, the control group showed shorter latency responding to the DS than did the experimental group, and the difference lasted throughout Test Day 1 . This is thus a case of negative transfer of control. The reason was that the experimental and control animals brought two different response repertoires to the test situation. The experimental animals had learned a FCAR to tone. But the FCAR, not part of the bar- 
press response sequence, was a competing response during the test phase. The control group, without this competing response, was unhindered. The data support our analysis that instrumental response mediators may be part of a necessary response sequence and enhance or detract from performance.

As for the data from the entire test phase, the decrease in the mean latency from Day 1 to Day 2 for the experimental group (see Figure 2) appeared to be reflecting two processes. First, the animals continued to acquire the responses of pressing the bar and approaching the food cup, each one tending to elicit the other. As this learning occurred, the FCAR entered into a functional sequence. Second, the prolonged "head-in-the-cup" behavior extinguished, as in Experiment 1. The barpress response sequence to the food reinforcer also became established for the control animals, but attending to the tone and the FCAR to the tone had not been learned. Thus, their improvement was not as great. This is consistent with the behavioral observation made in Experiment 1, in which time between tone onset and the FCAR was very variable for the control group during the entire test phase.

The present analysis was not designed to deal with questions concerning any noninstrumental response-mediator effect. The point is that the data suggest that the experimental procedure used here, and similar ones, cannot be employed to answer unambiguously any questions related to the mediator characteristics. Thus, although Trapold and Overmier (1972) assert that "something else gets learned during the pairing stage of transfer experiments over and above any peripheral operants" (p. 437), such an assertion does not appear justified in the appetitive transfer experiment. Because transfer experiments have not controlled for the occurrence of various instrumental responses previously learned by the experimental but not the control animals, it is not possible thereby to infer the presence of a noninstrumental response mediator. It is evident that a better experimental procedure is needed before one can start to answer questions about the mediator characteristics. Moreover, more generally, the results suggest that animal learning situations may require more detailed behavioral analysis than is sometimes accorded them and that observations of behavior other than that of a simple criterion response may be informative con- cerning the process involved. Centrally, the more detailed analysis of the transfer situation suggests on a theoretical level that negative transfer can occur, and the present results give support to that expectation.

\section{REFERENCE NOTE}

1. Overmier, J. B., \& Lawry, J. A. Pavlovian conditioning and the mediation of behavior. Unpublished manuscript, 1979.

\section{REFERENCES}

Carlson, J. G. Effect of a stimulus paired with reinforcement as a function of reinforcement magnitude. Bulletin of the Psychonomic Society, 1976, 7, 254-256.

Flaherty, C. F., \& Davenport, F. W. Noncontingent pretraining in instrumental discrimination between amounts of reinforcement. Journal of Comparative and Physiological Psychology, 1968, 66, 707-711.

Rescorla, R. A., \& Solomon, R. L. Two-process learning theory: Relationships between Pavlovian conditioning and instrumental learning. Psychological Review, 1967, 74, 151-182.

StaAts, A. W. A case in and strategy for the extension of learning principles to problems of human behavior. In A. W. Staats (Ed.), Human learning. New York: Holt, Rinehart, \& Winston, 1964.

StaAts, A. W. Learning, language, and cognition. New York: Holt, Rinehart, \& Winston, 1968.

StaAts, A. W. Social behaviorism. Homewood, Ill: Dorsey, 1975.

StaAts, A. W., \& W ARren, D. R. Motivation and three-function learning: Food deprivation and approach-avoidance to food words. Journal of Experimental Psychology, 1974, 103, 1191-1199.

Trapold, M. A., \& Overmier, J. B. The second learning process in instrumental learning. In A. H. Black \& W. F. Prokasy (Eds.), Classical conditioning II. New York: AppletonCentury-Crofts, 1972.

(Received for publication March 8, 1980.)

\section{ERRATUM}

Thorne, B. M., \& Bracy, O. L. The effects of simultaneous and serial lesions of the olfactory bulbs on muricide, irritability, and open-field activity in LongEvans female rats. Bulletin of the Psychonomic Society, $1980,15,143-146$. The author credit for this article should have been as follows: B. Michael Thorne, Odie L. Bracy, and Stephen M. McNally. 